\title{
Self-motion sensation influenced by visual fixation
}

\author{
GABRIELLE KELLER and VOLKER HENN \\ Neurological Clinic, University Hospital, Zürich, Switzerland
}

\begin{abstract}
Subjects were exposed to angular decelerations of between 1 and $50 \mathrm{deg} / \mathrm{sec}^{2}(1)$ in total darkness, (2) in view of a dim subject-stationary fixation light, or (3) inside an illuminated subjectstationary striped cylinder (conflict stimulation). Vestibularly induced eye movements led to the oculogyral illusion of object motion. This phenomenon can be distinguished from that of the sensation of self-rotation. At the end of deceleration, the initial velocity of self-rotation sensation is similar during all three stimulus conditions, but is reduced in duration with the conflict stimulus. Differences of interpretation in the literature concerning these phenomena can be explained on the basis of the failure to distinguish between the oculogyral illusion and sensation of self-motion and the inability to fully suppress vestibular eye movements.
\end{abstract}

In primates, extensive single-neuron recordings in the vestibular system have led to certain hypotheses about the neuronal basis of visual-vestibular interaction (for a review, see Henn, Cohen, \& Young, 1980 ). Once it was established that a visual stimulus could influence vestibular nuclei activity, the search began for an exact characterization of the visual parameters to convey such an influence. The experiments described below were first performed with single-neuron recordings in monkeys (Buettner \& Büttner, 1979; W. Waespe \& Henn, 1978). It was found that a visual stimulus accelerated in the same direction as the animal could influence vestibular nucleus activity in a very specific way. This influence depended on parameters of accelerations, velocities, and presentation of the visual stimuli. Specifically, if the visual stimulus was a small fixation light at which the animal was trained to look, the time constant of vestibular nuclei activity in response to a velocity step was shorter than the same vestibular stimulus would be in total darkness. If the animal was rotated with a subject-stationary (i.e., stationary with respect to the subject) surround, then peaks of vestibular neuron activity were attenuated. Correspondingly, the results of a study with human subjects, which explored subjective sensation during vestibular stimulation in darkness with a subject-stationary surround and using the same primate turntable, were compatible with predictions derived from animal experiments. Other studies, however, came to the conclusion that the presence of a subject-stationary visual object enhanced motion sensation instead of attenuating or shortening it (Huang \& Young, 1981; Marshall, 1967). We therefore undertook the investigation described below. We tried to perform the human ex-

The authors' mailing address is: Neurological Clinic, University Hospital, CH-8091 Zürich, Switzerland. periments under conditions that were as similar as possible to those used with monkeys. A common parameter of comparison is nystagmus. In the monkey, however, single neuron activity in the vestibular system can be measured, whereas in humans subjective motion sensation is described verbally.

\section{METHOD}

Fourteen healthy young adults (21-39 years old) participated; four of them repeated the test series four times. Ten of these subjects were experimentally naive; the other four had previously participated in various vestibular testing procedures.

The subjects were seated on a servocontrolled turntable that could be rotated about a vertical axis. A vertically striped cylinder (diameter $150 \mathrm{~cm}$, height $200 \mathrm{~cm}$, black and white stripes, each $7.5 \mathrm{deg}$ wide) totally enclosed the subject seated on the turntable. This cylinder could be driven by another servocontrolled motor or could be mechanically coupled to the turntable. The cylinder could be used as a lightproof enclosure or could be illuminated from within during full-field visual stimulation. A small light spot in the otherwise dark cylinder, positioned straight ahead of the subject and subtending over $.8 \mathrm{deg}$, could serve as a fixation point.

The subjects were rotated in random sequence under three experimental conditions of angular deceleration: in total darkness, with a dimly lit fixation light in front of the subject, and with the optokinetic drum mechanically coupled to the turntable and fully lit from within. The subjects were first accelerated and rotated at a constant angular velocity between 20 and $200 \mathrm{deg} / \mathrm{sec}$. They were then decelerated at $1 \mathrm{deg} / \mathrm{sec}^{2}$ (from $20 \mathrm{deg} / \mathrm{sec}$ ), $5 \mathrm{deg} / \mathrm{sec}^{2}$ (from $100 \mathrm{deg} / \mathrm{sec}$ ), and $10 \mathrm{or} 50 \mathrm{deg} / \mathrm{sec}^{2}$ (from $200 \mathrm{deg} / \mathrm{sec}$ ). Meusurements were taken only during decelerations. During acceleration, some motor noise was unavoidable and could have influenced sensation. During deceleration, this factor was much smaller and unnoticed by most subjects. If the fixation light was on, the subjects were asked to fixate it; during conflict stimulation, they were asked to look straight ahead at the stripes.

After preliminary test runs, a stimulus range of deceleration values between 1 and $50 \mathrm{deg} / \mathrm{sec}^{2}$ was chosen. Values lower than $1 \mathrm{deg} / \mathrm{sec}^{2}$ entered the threshold region for vestibular stimuli in some subjects, producing a large response variation and therefore making measurements unreliable. Values greater than $50 \mathrm{deg} /$ sec $^{2}$ during conflict stimulation are usually experienced as unpleasant, at times leading to nausea and motion sickness. There 
are no safety standards for prolonged high accelerations or high rotational velocities. Therefore, we limited velocities to $200 \mathrm{deg} / \mathrm{sec}$.

The subjects indicated motion sensation by rotating a handle fixed to the shaft of a potentiometer. Changes of position were indicated continuously by movement of the handle through a corresponding angle. One full subjective rotation was indicated by a full revolution of the handle. When added together, the revolutions give a measure of total amplitude of subjective motion. The subjects indicated any displacement of the visual field by pressing a button during the time that visual displacement was experienced. In addition, verbal comments through an intercom system were protocolled.

Horizontal eye position was measured by placing silver-silver chloride electrodes at the outer canthi of the eyes. Input signals were led through filters with a time constant of $3 \mathrm{sec}$. Directcurrent recording was halted after preliminary tests, because, during the conflict stimulation, many subjects sweated profusely, making stable dc recording impossible. Eye movements were calibrated by having the subjects look alternately at horizontally separated fixation lights. Resolution was about 1 deg.

The total duration of one full session for a subject was $2 \mathrm{~h}$. The subject's head rested comfortably against an adjustable support similar to that of a dentist's chair. He or she was asked not to move his or her head, and to press it slightly against the support. The subject was kept alert by the continuous tasks to be performed. If decreased vigilance was indicated by a deteriorating response, an adequate rest period was intercalated or the experiment was continued on another day.

Subjective position change, eye position, subjective visual field displacement, turntable position, and acceleration were recorded on a 6-channel rectilinear oscillograph with a paper speed of $10 \mathrm{~mm} / \mathrm{sec}$. All further measurements were made from these paper charts. In some experiments, these parameters were also recorded on an FM-tape machine for later reproduction.

\section{RESULTS}

An original record is shown in Figure 1 for the three different experimental conditions: rotation in darkness (A), and how the response to the same rotation is modified by viewing a fixation light (B) or by viewing the subject-stationary optokinetic drum (C). Presence of a visual stimulus greatly reduces nystagmus, but cannot fully suppress it at high decelerations, such as $50 \mathrm{deg} / \mathrm{sec}^{2}$. Only at decelerations below $5 \mathrm{deg} / \mathrm{sec}^{2}$ can nystagmus usually be fully suppressed. The presence of a subject-stationary visual stimulus also reduces the duration of the sensation of selfrotation. In addition to the experience of selfrotation, most subjects indicate that they perceive the visual image as moving (im).

Qualitatively, all subjects gave similar results: notably, responses from the 10 subjects who were experimentally naive and sat on a turntable for the first time did not differ from those of the four members of the laboratory who had experienced many hours of previous psychophysical testing. Furthermore, there was no difference in results between subjects who underwent only one test series and those who were tested repeatedly. These conclusions were based on comparisons of averages of single measurements and their standard deviations. Therefore, once statistics showed that these subgroups belonged to a single population, all data were pooled. In the subsequent

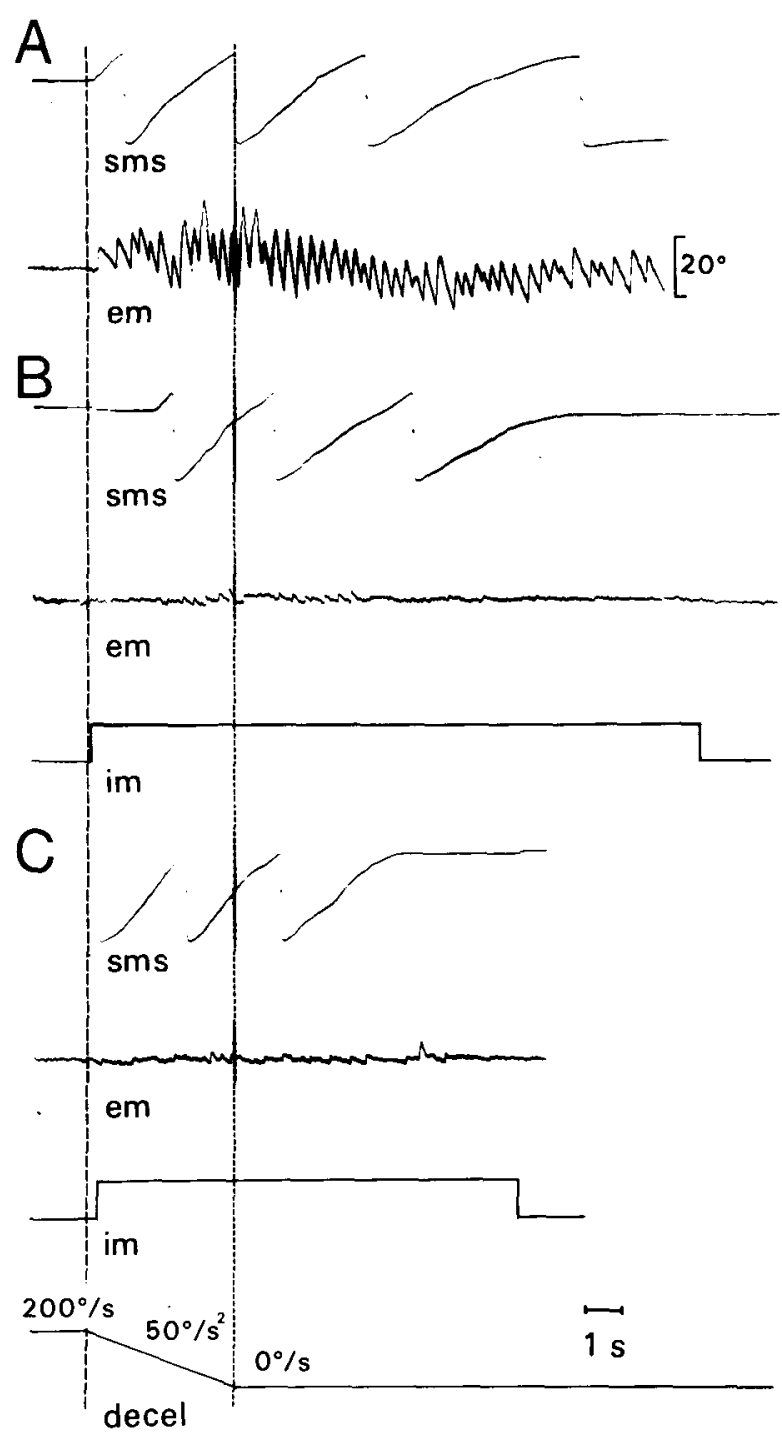

Figure 1. Original records of vestibular stimulation only (A), with a fixation light (B), and with a subject-stationary visual field (C). Abbrevintions: subjective position, sms; horkontal eye position, em; the occurrence of illusory image motion, im; decelernton, decel. With so-deg/sece deceleration, a visual stimulus reduces motion sensation only slightly, but nystagmus is much more attenuated.

description of results, only averages and standard deviations of the normalized data will be given.

Duration of subjective motion sensation and nystagmus after pulses of angular deceleration can vary widely. Instead of the total duration, time constants are usually given which vary at least between 10 and $30 \mathrm{sec}$ in normals. This variation has been interpreted as resulting from different states of habituation (Jaeger \& Henn, 1981). Therefore, all results are normalized, in the sense that they are given as a percentage of responses to the same deceleration in total darkness, either immediately before or immediately after the test run. 


\section{Subjective Motion Sensation}

Three parameters were measured: duration, total amplitude, and maximal velocity (Figure 2). Direction of motion sensation was always in the expected direction, that is, opposite to the previous direction of rotation. Each parameter tended to be lower during conflict than during vestibular stimulation in darkness (set at $100 \%$ ) or with the same deceleration but only the fixation light visible. At $1 \mathrm{deg} / \mathrm{sec}^{2} \mathrm{de}-$ celeration, 8 of the 14 subjects failed to sense the changing velocity during the conflict condition. Those trials are not included in the curves of Figure 2. At $5 \mathrm{deg} / \mathrm{sec}^{2}$ and above, all subjects had quantitatively similar responses. During conflict stimulation, the duration as well as the total amplitude of self-rotation was reduced to about $60 \%$.

A

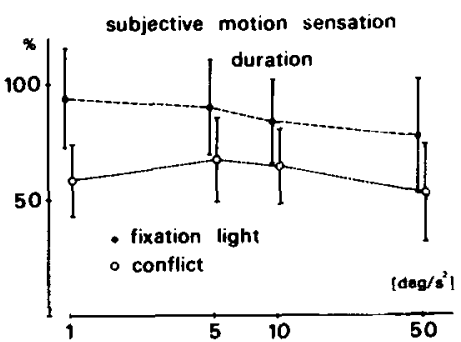

B
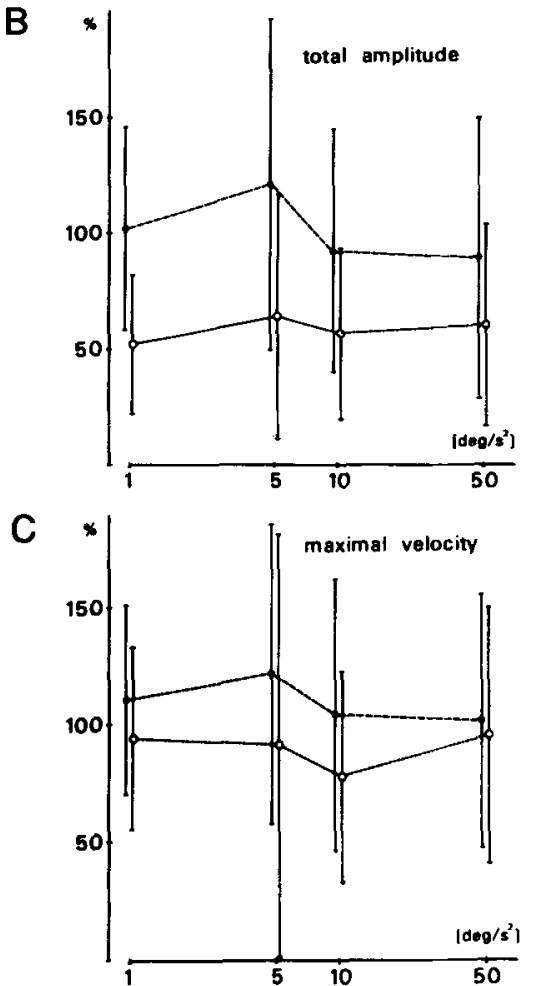

Flgure 2. Influence of a subject-stationary visual stimulus on perception of self-rotation relative to angular deceleration in darkness, plotted for different values of deceleration on a log scale. Single values were normalized to $100 \%$ (decelention without visual stimulus), from which the total average was calculated and used for this figure.
Within the range of stimulation, this was independent of the value of deceleration. Maximal velocity, usually occurring immediately following the end of deceleration, was less affected.

During deceleration with the fixation light visible, motion sensation was shortened, but much less so than with the full-field stationary pattern. Total amplitude and maximal velocity at $10-\mathrm{deg} / \mathrm{sec}^{2}$ deceleration and values above were not altered on average. At lower values, there was a tendency for these parameters to be slightly enhanced.

\section{Eye Movements}

Eye movements were suppressed in similar amounts under the two conditions of a visual stimulus. With 1-deg/ $\mathrm{sec}^{2}$ deceleration, electro-oculographic records indicated no eye movement, although many subjects experienced the deceleration. Because of the ac recording, slow tonic deviations could not be detected, and so the measurements were restricted to latency (start of deceleration to the first occurrence of a rapid nystagmus phase, Figure $3 \mathrm{C}$ ), the interval between start of deceleration and the occurrence of maximal eye velocity (Figure $3 B$ ), and the value of maximal eye velocity (Figure 3A). As nystagmus was greatly reduced, that is, much more so than the subjective motion sensation, the latency to the first rapid phase of nystagmus increased. The time of its maximal occurrence, measured as elapsed time after the start of deceleration, did not change. In general, the subjects were better able to suppress eye movements with the single fixation light than with full-field exposure.

\section{Percelved Motion of the Visual Stimulus}

Perceived motion of the visual stimulus could be differentiated from self-motion by all subjects. If that distinction was not reported spontaneously, the subjects were instructed to consider as self-motion only that sensation which they also experienced during acceleration in total darkness. Motion of the fixation light at low decelerations was described as a slow displacement in the direction of the self-motion sensation, or, if self-motion was not perceived, in the expected direction. At high decelerations, it was described as an oscillating to-and-fro motion, tending to elongate the fixation point to a line, again in the direction of the self-motion sensation.

With full visual field stimulation, the vertical bars seemed to move in a horizontal direction to either side, paradoxically without changing their position. The motion sensation was most pronounced in the center, leading to a curvature of the vertically aligned stripes of the drum. At higher decelerations, the whole visual image became unstable, comparable to a jitter. This coincided with the occurrence of nystagmus.

At the lowest decelerations, the illusory motion was shorter than the duration of the deceleration 

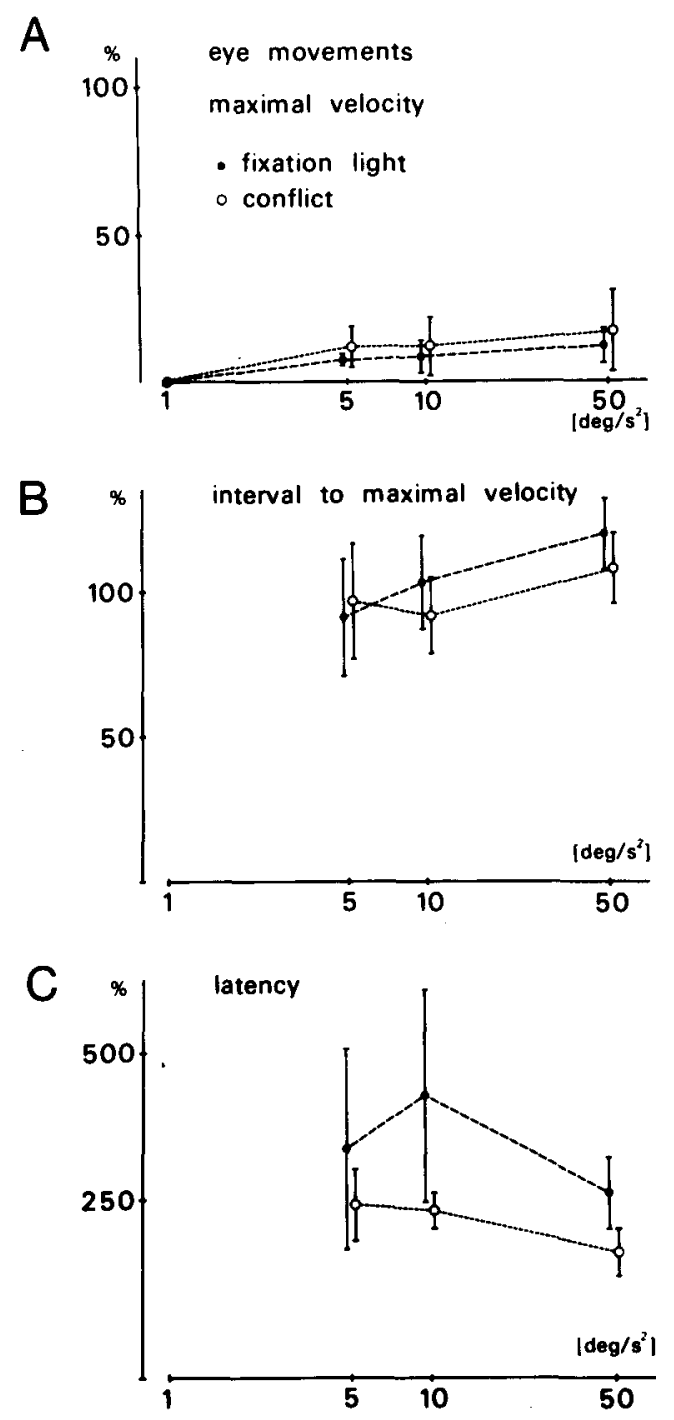

Figure 3. Change in eye-movement parameters relative to deceleration in darkness, set at $\mathbf{1 0 0 \%}$. Eye movements were more attenuated with the fixation light only than with the full visual field. Single values were first normalized and then pooled to calculated averages.

stimulus (Figure 4) and, on average, shorter than the self-motion sensation (Figure 5). At high decelerations, the values showed the opposite relationship. Absolute duration was longer, and visual image motion became longer than the sensation of self-motion.

\section{Comparison of Subjective Motion Sensation, Visual Image Motion, and Nystagmus}

In the dark, with an angular deceleration stimulus only, nystagmus usually outlasts subjective motion sensation. In the presence of a visual stimulus, this relation reverses. Eye movements are suppressed to a much greater degree than the sensation of motion. Visual phenomena of object motion are two kinds, a slow lateral deviation of the image or a jitter that leads to blurring of the image. Although a lateral deviation can be observed even at the lowest decelerations, the jitter was seen only at high decelerations, and it always occurred together with nystagmus.

\section{DISCUSSION}

The experiments gave the clear result that a subjectstationary visual stimulus influences motion sensation. Variables such as ranges of acceleration, the subjects' ability to suppress eye movements, the instructions to the subjects, and the nature of the visual stimulus are relevant parameters that are important for interpretation of the mechanisms.

Attempted fixation of a small light during deceleration has less influence on the perception of selfmotion than does whole-field illumination. Compared with deceleration in total darkness, fixation of a light spot tends to reduce the overall duration of motion sensation at high decelerations (Figure 2). Eye movements are suppressed to a much greater degree, although not totally. Subjects experience a lateral deviation of the fixation light, known as the oculogyral illusion (Graybiel \& Hupp, 1946). Although our subjects could clearly distinguish between illusory object and self-motion, the two obviously influence each other. At low decelerations, the oculogyral illusion enhances the velocity of motion sensation (Clark \&

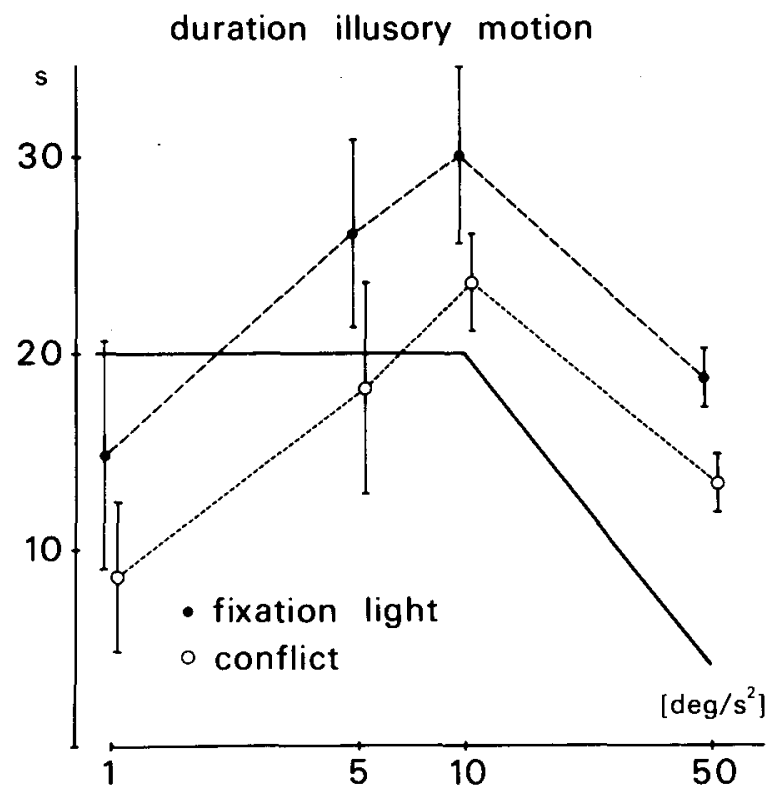

Figure 4. The duration of lllusory motion in absolute values. The solid line indicates the duration of the deceleration stimulus, which was 20 sec for decelerations between 1 and $10 \mathrm{deg} / \mathrm{sec}^{2}$ and 4 sec for decelerations of $50 \mathrm{deg} / \mathrm{sec}^{2}$. In the conflict situation, at 1$\mathrm{deg} / \mathrm{sec}^{2}$ deceleration, the lllusory motion lasts for about half the duration of the decelerition, and at 50-deg/ $/ \mathrm{sec}^{2}$ deceleration, it is about three times as long. With the fixation light only, all values are longer. 


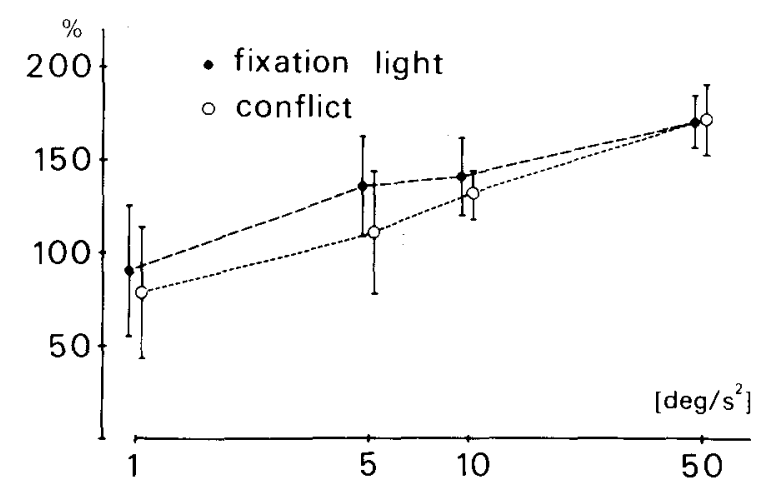

Figure 5. The ratio between the durations of illusory object motion and self-motion. At $\mathbf{1 0 0 \%}$ illusory motion and self-motion have the same duration. Except for the lowest deceleration values, object motion is usually perceived to be longer.

Stewart, 1969). At high decelerations, an oscillatory motion of the visual stimulus is superimposed on a lateral deviation, always together with the occurrence of nystagmus. As the subject tries to fixate the light, he is successful in markedly reducing the strength of nystagmus (Figure 3). The sensation of self-motion is slightly reduced in its duration (Figure 2) and usually is outlasted by the oculogyral illusion (Figure 5). We interpret these opposing tendencies in such a way that any additional motion clue, such as the oculogyral illusion, enhances motion sensation at deceleration values near the vestibular threshold, when stimuli might be ambiguous. At high decelerations, when the vestibular stimulus is unambiguous, any subjectstationary stimulus tends to decrease motion sensation, although the oculogyral illusion is still present.

The laboratory observations described above can be interpreted in terms of the real-life condition of head-turning during visual pursuit of a moving target. In that situation, the self-motion sensation should be preserved unaltered whether the vestibuloocular reflex moves the eyes in the direction opposite to that of a head turn, or whether the eyes move in the direction of the visual target. In the range of most combined eye-head movements that are either slow or of very short duration, self-motion perception does not alter depending on eye movements, as our experiments confirm.

Rotation in a subject-stationary environment is experienced as totally different, because it creates a sensory conflict: during deceleration, the labyrinth signals an impulse of deceleration, whereas the visual system conveys the message that no motion is taking place. In most subjects, during either long duration or high values of deceleration, this is experienced as unpleasant, if not overtly leading to motion sickness with profuse sweating and nausea.

The whole-field visual stimulus has the effect of shortening the duration of the motion sensation but has less effect on the perception of initial maximal ve- locity. Again, eye movements are suppressed to a considerable extent, but less so than when the foveal stimulus alone is used. This is unexpected and cannot be readily explained. One possibility is that the sensory conflict detracts from performance of the task. Again, illusory object motion that is clearly distinguishable from self-motion is reported. The nature of object motion is similar to that of a fixation light: first the stripes are drawn to the side; later, if nystagmus occurs, an oscillatory motion is superimposed. Some subjects then make the further differentiation that they see a continuous motion of the stripes in one direction without a change in position. This constitutes a logical paradox. This contradiction between continuing motion with stable position has been reported for other forms of visual-vestibular interaction, for example, roll vection induced by a rotating disk (Held, Dichgans, \& Bauer, 1975).

Few studies have investigated the influence of a visual stimulus on the sensation of motion, although the purely visually induced sensation of self-motion has been investigated in detail (Brandt, Dichgans, \& Koenig, 1973; Dichgans \& Brandt, 1978; Fischer \& Kornmüller, 1930; Mach, 1875/1967). Marshall (1967) compared the subjective threshold for angular acceleration during rotation in the dark and with a lighted subject-stationary visual field. Accelerations were between 1.5 and $12 \mathrm{deg} / \mathrm{sec}^{2}$. The subject was asked to close a switch "to indicate his awareness of movement." At $1.5-\mathrm{deg} / \mathrm{sec}^{2}$ acceleration, the average latency decreased from $12 \mathrm{sec}$ in the dark to $8 \mathrm{sec}$ in the lighted condition, although in $7 \%$ of the trials subjects reported no sensation of acceleration at all. With higher accelerations, this effect becomes smaller and is nonsignificant at $12 \mathrm{deg} / \mathrm{sec}^{2}$. Our interpretation of these data is that the oculogyral illusion led to an earlier "awareness of movement" at low accelerations, as in our experiments.

Huang and Young (1981) did a more extensive study. Their experiments were performed in an aircraft trainer with velocities limited to $40 \mathrm{deg} / \mathrm{sec}$ and accelerations limited to $20 \mathrm{deg} / \mathrm{sec}^{2}$. A further difference between their study and ours is that they did not measure eye movements or require from the subject a distinction between object and self-motion. They concluded that "detection and reaction to angular acceleration" was enhanced in the presence of a stationary visual input at low accelerations. In interpreting their data, they state that "the oculogyral illusion has a greater facilitating effect on the detection of self-rotation at low accelerations than any inhibitory effect of the striped fixed visual field" (p. 180). Their apparatus did not allow for the testing of greater prolonged accelerations.

These comparisons imply that phenomena in effect at accelerations near the vestibular threshold are different from those in effect at accelerations of greater values. Taking into account the distinction between 
the oculogyral illusion and the sensation of selfmotion, as compared using the global statement "awareness of movement" (Huang \& Young, 1981; Marshall, 1967), the above two reports and a further one from our laboratory (B. Waespe, W. Waespe, \& Henn, 1980) actually agree that there are two opposing factors of how a visual stimulus influences motion detection: the oculogyral illusion can lead to an enhanced awareness of motion, especially at low accelerations, but the sensation of self-rotation is reduced, especially at high accelerations.

The resolution and ac recording of eye movements used in our experiments cannot contribute to the discussion about the relationship between the oculogyral illusion and eye movement. Arguments have been proposed to show that the oculogyral illusion can occur in the absence of measurable eye movements (Byford, 1963; van Dishoeck, Spoor, \& Nijhoff, 1954; Vogelsang, 1961), or it has been suggested that it might be caused by internal oculomotor signals without effecting eye movements (Whiteside, Graybiel, \& Niven, 1965). On the other hand, after using higher resolution eye-movement recording, the question of how completely eye movements can actually be suppressed during vestibular stimulation has been raised (Barnes, 1983).

\section{Animal Experiments}

Monkeys have been exposed to the same stimulus conditions, with eye movements and single-neuron activity measured in the vestibular nerve and nuclei. Animals were trained to fixate an animal-stationary light spot while being rotated about a vertical axis. For investigation of the conflict stimulus, most animals were untrained.

As in the human case, animals can suppress nystagmus during low decelerations but are less successful the higher these values become. Single-neuron activity in the nerve is not influenced by any visual stimulus or behavioral task (Büttner \& W. Waespe, 1981). Activity in the vestibular nuclei is profoundly influenced by visual stimuli. During conflict stimulation, the time constant of activity is always shortened. The maximal activity of neurons is strongly reduced during low decelerations, but to a much lesser extent during higher values, that is, above $10-20 \mathrm{deg} / \mathrm{sec}^{2}$ (W. Waespe \& Henn, 1978). In the human case, there is also no difference for initial velocity perception at high decelerations, but the duration of self-motion sensation is shortened.

With a fixation light, single-neuron activity in the vestibular nuclei during deceleration leads to unaltered initial activation during the deceleration, but time constants are shortened (Buettner \& Büttner, 1979). Compared with humans, there is similar initial perception of velocity, which is shortened in its duration, but less so when compared with the conflict situation.

\section{Conclusion}

There are many differences between monkey and man in terms of the vestibular time constant and other quantitative parameters of visual-vestibular interactions (Cohen, Henn, Raphan, \& Denett, 1981). Still, it is astonishing to what high degree there is a correspondence between human subjective response and single-neuron recordings in the vestibular nuclei of monkeys, although these structures are only the first stage of sensory convergence projecting to the thalamus and parietal cortex. Like others, we have shown that a visual stimulus influences vestibular perception, have further specified quantitative parameters, and have determined to what extent the perception of the vestibular stimulus is modified by its own reflex actions such as the oculogyral illusion and nystagmus.

\section{REFERENCES}

Barnes, G. R. (1983). The effects of retinal target location on suppression of the vestibulo-ocular reflex. Experimental Brain Research, 49, 257-268.

Brandt, Th., Dichgans, J., \& Koenig, E. (1973). Differential effects of central versus peripheral vision on egocentric and exocentric motion perception. Experimental Brain Research, 16, 476-491.

Buettner, U. W., \& Büttner, U. (1979). Vestibular nuclei activity in the alert monkey during suppression of vestibular and optokinetic nystagmus. Experimental Brain Research, 37, 581-593.

BütTNer, U., \& WAEspe, W. (1981). Vestibular nerve activity in the alert monkey during vestibular and optokinetic nystagmus. Experimental Brain Research, 41, 310-315.

Byford, G. H. (1963). Eye movement and the optogyral illusion. Aerospace Medicine, 34, 119-123.

Clark, B., \& Stewart, J. D. (1969). Effects of angular acceleration on man: Thresholds for the perception of rotation and the oculogyral illusion. Aerospace Medicine, 40, 952-956.

Cohen, B., Henn, V., Raphan, T., \& DenetT, D. (1981). Role of velocity storage in visual-vestibular interactions in humans. Annals of the New York Academy of Sciences, 374, 421-433.

Dichoans, J., \& Brandt, TH. (1978). Visual-vestibular interaction: Effects on self-motion perception and postural control. In R. Held, H. Leibowitz, \& H. L. Teuber (Eds.), Handbook of sensory physiology (Vol. 8, pp. 755-804). New York: Springer.

Fischen, M. H., \& KonnmÚllez, A. E. (1930). Optokinetisch ausgelöste Bewegungswahrnehmungen und optokinetischer Nystagmus. Journal für Psychologie und Neurologie, 41, 273-308.

Graybiel, A., \& HuPP, D. I. (1946). The oculogyral illusion; a form of apparent motion which may be observed following stimulation of the semicircular canals. Journal of Aviation Medicine, 17, 3-27.

Held, R., Dichoans, J., \& Bauer, J. (1975). Characteristics of moving visual scenes influencing spatial orientation. Vision Research, 15, 357-365.

Henn, V., Cohen, B., \& Young, L. R. (1980). Visual-vestibular interaction in motion perception and the generation of nystagmus. Neurosciences Research Program Bulletin, 18, 457-651.

Huang, J., \& Young, L. R. (1981). Sensation of rotation about a vertical axis with a fixed visual field in different illuminations and in the dark. Experimental Brain Research, 41, 172-183.

JAEGER, J., \& HeNN, V. (1981). Vestibular habituation in man and monkey during sinusoidal rotation. Annals of the New York Academy of Sciences, 374, 330-339.

Mach, E. (1967). Grundlinien der Lehre von den Bewegungs- 
empfindungen. Amsterdam: Bonset, 1967. (original work published 1875)

Marshall, J. E. (1967). Visual-vestibular interaction and threshold for angular acceleration (Report 754). Fort Knox, KY: U.S. Army Medical Research Laboratory.

van Dishoeck, H. A. E., Spoon, A., \& Nijhoff, P. (1954). The opto-gyral illusion and its relation to the nystagmus of the eyes. Acta Otolaryngologica, 44, 597-607.

Vogelsana, C. J. (1961). The perception of a visual object during stimulation of the vestibular system. Acta Otolaryngologica, 53, $461-469$.
WAEspe, B., WAEspe, W., \& HenN, V. (1980). Subjective velocity estimation during conflicting visual-vestibular stimulation. Archiv für Psychiatrie und Nervenkrankheiten, 228, 109-116.

WAEsPE, W., \& HENN, V. (1978). Conflicting visual-vestibular stimulation and vestibular nucleus activity in alert monkeys. Experimental Brain Research, 203-211.

WAEsPe, W., \& HENN, V. (1979). The velocity response of vestibular nucleus neurons during vestibular, visual, and combined angular acceleration. Experimental Brain Research, 37, 337-347.

Whiteside, T. C. D., Graybiel, A., \& Niven, J. I. (1965). Visual illusions movement. Brain, 88, 193-210.

(Manuscript received June 7, 1983;

revision accepted for publication January $12,1984$. 